\title{
Phase Offset of Rotation-Powered X-ray Pulsars
}

\author{
H. G. Wang, G. J. Qiao, and R. X. Xu \\ Department of Astronomy, Peking University, 100871, China
}

\begin{abstract}
Combing the data of all the 10 rotation-powered pulsars that have measurements of relative phase of radio and X-ray pulses, we report here the behavior of radio/X-ray phase offset. We find that the radio/X-ray offset of normal pulsars does not show the trend of increase with increasing pulsar period as the radio/gamma-ray offset exhibits. The offset of millisecond pulsars is generally much less than that of the normal pulsars. Such phenomenon should also be taken into account by the high energy models for pulsars.
\end{abstract}

The phase offset between pulsar radio and high energy pulses is very helpful for us to understand the high energy emission. Base on 6 gamma-ray pulsars, some authors found that the radio/gamma-ray phase offset increase with increasing pulsar period (Ulmer 1994). Here we investigate the behavior of radio$\mathrm{X}$ phase offset by combing the data of all the pulsars of which the relative phase of radio and $\mathrm{X}$-ray pulses has been measured.

In principle we define the radio/X-ray offset as the phase separation between the first radio and the first X-ray peaks. The offset is chosen so that its value is less than 0.5. For PSR J0437-4715, J0218+4232 and B1706-44 this method is no longer valid because of the complexity of their radio profiles. In these cases, noticing that the X-ray pulses appear to be well aligned with their radio pulses, we make specific measurement for each but do not affect the general picture of alignment. When no estimate of phase offset is given in literature, we measure it by hand, with taking one X-ray phase bin as the error.

The information is summarized in Table 1 and the data are plotted in Fig.1. It shows that, for normal pulsars, the radio/X offset does not show the trend as the radio/gamma offset exhibits, namely, to increase with increasing pulsar period. The offset of millisecond pulsars is generally much less than the former, which may be due to the smaller radius of the light cylinder. We suggest that this phenomenon, together with the behavior of radio/gamma offset, need to be fully considered by any high energy model or 'united' model for pulsars.

Acknowledgments: This work is supported by National Nature Science Foundation of China (10173002), and by the Special Funds for Major State Basic Research Projects of China. 
Table 1. Radio-X phase offsets for 10 pulsars. The offsets measured by hand are marked with ' $\mathrm{H}$ '.

\begin{tabular}{llllll}
\hline \hline Pulsar & Period $(\mathrm{ms})$ & R-X offset & X-ray band (KeV) & comment & Ref. \\
\hline B1937+21 & 1.56 & $0.02 \pm 0.07$ & $1.7-6.5$ (ASCA) & & $\mathrm{T}+01$ \\
J0218+4232 & 2.32 & $0.07 \pm 0.09$ & $0.08-10$ (Chandra) & $\mathrm{H}$ & $\mathrm{K}+02$ \\
B1821-24 & 3.05 & $0.02 \pm 0.006$ & $2-16$ (RXTE) & & $\mathrm{R}+98$ \\
J0437-45 & 5.76 & $0.03 \pm 0.04$ & $0.08-10$ (Chandra) & & $\mathrm{Z}+02$ \\
B0531+21 & 33.52 & 0.05 & $0.5-2.4$ (ROSAT) & $\mathrm{H}$ & $\mathrm{T} 01$ \\
J1420-6048 & 68.2 & $0.25 \pm 0.1$ & $1-10$ (ASCA) & $\mathrm{H}$ & $\mathrm{R}+01$ \\
B0833-45 & 89.33 & $0.23 \pm 0.05$ & $0.1-1.5$ (ROSAT) & $\mathrm{H}$ & $\mathrm{HM} 98$ \\
B1706-44 & 102.5 & $0.05 \pm 0.1$ & $0.1-10$ (Chandra) & $\mathrm{H}$ & $\mathrm{G}+02$ \\
B1509-58 & 150.7 & $0.27 \pm 0.01$ & $2-16$ (RXTE) & & $\mathrm{R}+98$ \\
B1055-52 & 197.1 & $0.20 \pm 0.05$ & $0.5-2.4$ (ROSAT) & $\mathrm{H}$ & $\mathrm{T}+99$ \\
\hline \hline
\end{tabular}

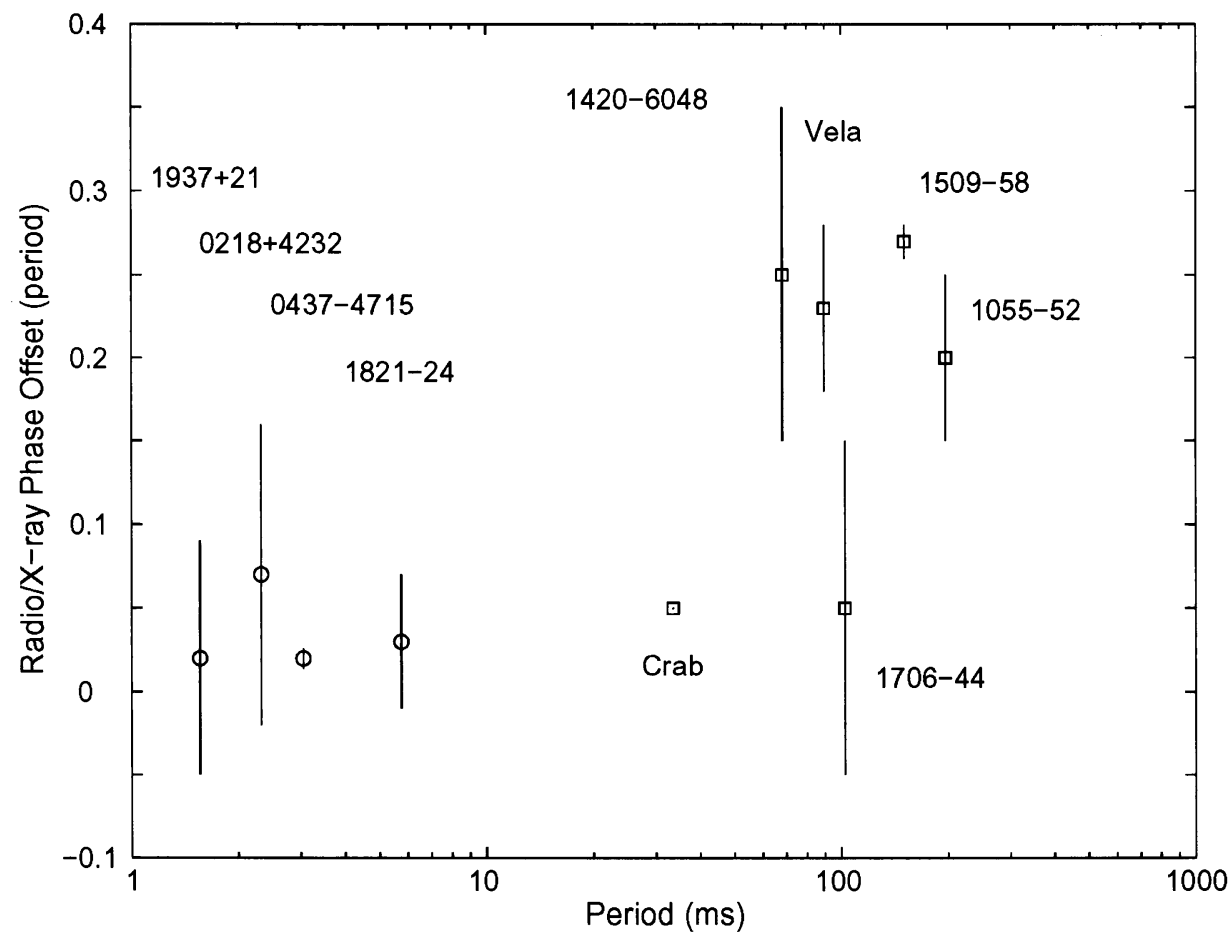

Figure 1. Plot of the radio-X-ray phase offsets vs. pulsar period, with squares for normal pulsars and circles for millisecon pulsars. 


\section{References}

Gotthelf, E. V., Halpern, J. P. \& Dodson, R, 2002, ApJ, 567, L125 (G+02)

Harding A.K. \& Muslimov A.G., 1998, ApJ, 500, 862 (HM98)

Kuiper, L., Hermsen, W., Verbunt, F. et al., 2002, ApJ, 577, 917 (K+02)

Roberts, M., Romani, R.W. \& Johnston, S., 2001, ApJ, 561, L187 (R+01)

Rots A.H., Jahoda K., Macomb D.J. et al., 1998,ApJ, 501, 749 (R+98)

Takahashi, M. et al., 2001, ApJ, 554, 316 (T+01)

Thompson D.J. et al., 1999, ApJ, 516, $297(\mathrm{~T}+99)$

Thompson,D.J., 2001, in High Energy Gamma-Ray Astronomy, eds. F. A. Aharonian and H.J. Volk, AIP Conference Proceedings, 558, 103 (T01)

Ulmer M.P., 1994, ApJ, 90, 789

Zavlin, V. E., Pavlov, G. G., Sanwal, D. et al., 2002, ApJ, 569, 894 (Z+02) 